\title{
Compensation of Impedance Meters When Using an External Front-End Amplifier
}

\author{
Josep M. Torrents and Ramon Pallàs-Areny, Fellow, IEEE
}

\begin{abstract}
Four-terminal impedance meters based on pseudo-bridges yield unexpected uncertainties when using high-contact-impedance electrodes. Adding a front-end amplifier to the impedance meter and rearranging the connection of the meter terminals overcome the contact impedance problem. However, because the compensation provisions in the instrument are meant to compensate only impedance residuals of test fixtures, by either an open/short or an open/short/load correction procedure, the external front-end increases the inaccuracy of the measurement setup. This paper shows that an open/short/load correction can also compensate complicated impedance residuals such as those from external amplifiers. The paper details the correction procedure and provides the equations to calculate the impedance under test from the readings of the impedance meter.
\end{abstract}

Index Terms-Impedance measurement, pseudo-bridge, residual impedance, .

\section{INTRODUCTION}

$\mathbf{E}$ LECTRICAL impedance measurements, including impedance spectroscopy, have been applied to material characterization for sensor development and quality control in several areas ranging from biological tissue characterization [1] to cement setting studies [2]. Common impedance measurements need immersed electrodes when applied to ionic media [3] in order to convert ionic currents in the electrolyte into electron currents in electronic circuits. Electrode contact impedance is usually high, particularly at low frequencies. Because most impedance meters are mainly intended to characterize electronic components or circuits where contact impedances are low, using high-impedance electrodes causes the instrument to display inaccurate results.

Fig. 1 shows the basic working method for commercial impedance meters based upon pseudo-bridge techniques (auto-balance bridge circuit) such as the HP4192A [4]. The instrument applies a test signal of frequency $f_{s}$ and amplitude $V_{s}$ (selected by the user) to the impedance under test $\left(Z_{x}\right)$ through the high-current (HC) terminal, resulting in a current $I_{x}$ through $Z_{x}$ and a drop in voltage $\left(V_{x}\right)$ sensed at the high-potential (HP) terminal $\left(V_{\mathrm{HP}}=V_{x}\right)$.

Most of $I_{x}$ flows through an internal reference resistor $R_{r}$ toward a voltage-controlled voltage source $V_{r}$ locked in phase to the test signal and connected to the low-current (LC) ter-

Manuscript received April 3, 2001; revised December 31, 2001. This work was supported by the Spanish CICYT, under Project TAP1999-0742.

The authors are with the Divisió d'Instrumentació i Bioenginyeria, Departament d'Enginyeria Electrònica, Universitat Politècnica de Catalunya, Barcelona, Spain (e-mail: elerpa@eel.upc.es).

Publisher Item Identifier S 0018-9456(02)04313-9.

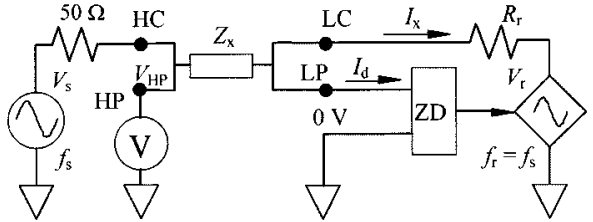

Fig. 1. Four-wire impedance measurement using a pseudo-bridge when contact impedances with the impedance under test $\left(Z_{x}\right)$ are negligible.

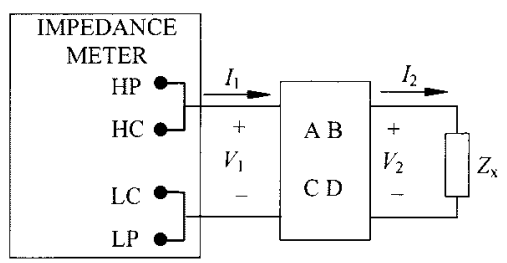

Fig. 2. Two-wire impedance measurement using a test fixture modeled by its $A B C D$ parameters.

minal. The remaining current, $I_{d}$, flows toward a zero detector (ZD) connected to the low-potential (LP) terminal. The output of the ZD controls the amplitude and phase of $V_{r}$ in order to achieve $I_{d}=0$ and a zero voltage at the LP terminal. Then, $Z_{x}=V_{\mathrm{HP}} / I_{x}$ and $I_{x}=-V_{r} / R_{r}$. Because $R_{r}$ is known, measuring $V_{\mathrm{HP}}$ and $V_{r}$ yields the value of the impedance under test, $Z_{x}$. Stray capacitance between the terminals of $Z_{x}$ and ground would not influence the measurement because terminals LP and LC are at zero potential and terminal HP is connected to a high-impedance voltmeter.

Nevertheless, the impedance under test is actually connected to the impedance meter by passive elements, such as port extensions and test fixtures, not shown in Fig. 1. In the simple two-wire connection, any inaccuracy attributable to passive elements, such as residual impedance of test fixtures, stray impedance, or admittance and connecting lead parasitics, can be compensated by two reference measurements at the test terminals, normally an open-circuit and a short-circuit impedance.

Any passive network connecting an impedance meter to the impedance under test by two wires (Fig. 2) can be represented by its transmission parameters $A, B, C$, and $D$ ([4], p. C-1). The input-output relation is then

$$
\left(\begin{array}{l}
V_{1} \\
I_{1}
\end{array}\right)=\left(\begin{array}{ll}
A & B \\
C & D
\end{array}\right)\left(\begin{array}{l}
V_{2} \\
I_{2}
\end{array}\right)
$$

where, by comparing with Fig. $1, V_{1}=V_{\mathrm{HP}}$ and $I_{1}=I_{x}+I_{d}$, if $I_{d}=0$ and $I_{1}=I_{x}$. 
The result for the measured impedance (meter reading) is $Z_{x m}=V_{1} / I_{1}$. Hence, we have

$$
Z_{x m}=\frac{V_{1}}{I_{1}}=\frac{A V_{2}+B I_{2}}{C V_{2}+D I_{2}} .
$$

The impedance under test (effective impedance, which includes parasitics and influence factors such as temperature and frequency) is $Z_{x}=V_{2} / I_{2}$. Therefore, $Z_{x}$ can be calculated from $Z_{x m}$ if the network transmission parameters $A$ to $D$ are determined from independent measurements. An open-circuit measurement $\left(I_{2}=0\right)$ yields $Z_{\mathrm{om}}=A / C$ and a short-circuit measurement $\left(V_{2}=0\right.$ ) yields $Z_{\mathrm{sm}}=B / D$. Then, if the measured network (which includes the instrument input port extension and the test fixture) is symmetric $(A=D)$, the actual impedance under test can be calculated from the measured impedance value by

$$
Z_{x}=\left(Z_{x m}-Z_{\mathrm{sm}}\right) \frac{Z_{\mathrm{om}}}{Z_{\mathrm{om}}-Z_{x m}}
$$

which agrees with the expression given by [4, p. C-2].

For complicated residuals such as those from custom-made test fixtures and external amplifiers, which may be asymmetric $(A \neq D)$, adding a third reference measurement involving an impedance close to the impedance under test further reduces inaccuracies [6]. This procedure is termed open/short/load correction. The final result is then

$$
Z_{x}=\left(Z_{x m}-Z_{\mathrm{sm}}\right) \frac{Z_{\mathrm{ref}}}{Z_{\mathrm{rm}}-Z_{\mathrm{sm}}} \frac{Z_{\mathrm{om}}-Z_{\mathrm{rm}}}{Z_{\mathrm{om}}-Z_{x m}}
$$

where $Z_{\text {ref }}$ is a standard or reference impedance and $Z_{\mathrm{rm}}$ is its measured value. Equation (4) is the arranged expression given by [6]. This procedure, however, is valid in principle only for two-wire measurements.

Moreover, if in Fig. 1 we consider the contact impedance $\left(Z_{e}\right)$ between the instrument and the impedance under test, we obtain the equivalent circuit in Fig. 3. The LC terminal is no longer at $0 \mathrm{~V}$, which results in gross measurement errors. An open/short/load correction, such as that discussed above, would not solve the problem because it only applies to two-wire measurements.

Adding a front-end differential amplifier (DA) with high input impedance to the impedance meter and rearranging its connections, as shown in Fig. 4, solve the high-contact impedance problem [5]. The voltage at the terminal HP is still the drop in voltage across the impedance under test because LC and LP are shorted and LP is at virtual ground potential. Therefore, the virtual ground at LC (LP, zero detector) yields $I_{x}=-V_{r} / R_{r}$ in spite of $Z_{e}$. However, any stray capacitance between the terminals of $Z_{x}$ and ground would influence the measurement because $Z_{x}$ is not virtually grounded.

Nevertheless, the front-end amplifier adds its own impedance residuals and the question arises about how to correct them. This paper shows an effective procedure and provides the necessary equations to reduce inaccuracies resulting from the front-end amplifier needed for impedance measurements involving highcontact impedances, such as those from immersed electrodes. The proposed procedure follows the same steps than that described for two-wire measurements.

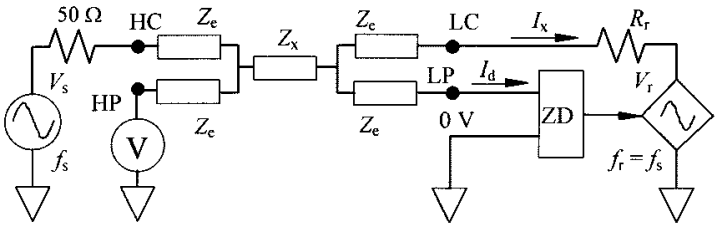

Fig. 3. Four-wire impedance measurement using a pseudo-bridge when contact (electrode) impedances with the impedance under test are not negligible.

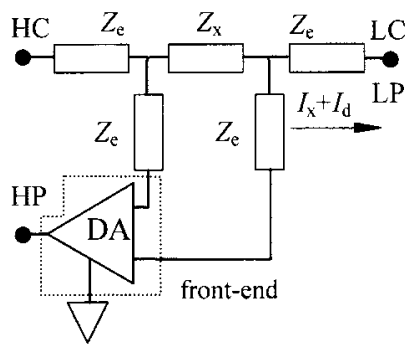

Fig. 4. Front-end amplifier (DA) and rearranged connections with the pseudo-bridge impedance meter in Fig. 3 as proposed in [5]. Because terminals LC and LP are shorted, the electrode impedance in series with the internal reference resistor $\left(R_{r}\right.$ in Fig. 3$)$ is at virtual ground potential.

\section{METHOD}

Any active circuit between the instrument and $Z_{x}$, such as the amplifier shown in Fig. 4, can be described by its transmission parameters (voltage gain $k$, input impedance $Z_{i}$, and output impedance $Z_{0}$ ) as follows

$$
\left(\begin{array}{c}
V_{V 1} \\
I_{V 1}
\end{array}\right)=\left(\begin{array}{ll}
A_{V} & B_{V} \\
C_{V} & D_{V}
\end{array}\right)\left(\begin{array}{c}
V_{V 2} \\
I_{V 2}
\end{array}\right)=\frac{1}{k}\left(\begin{array}{cc}
1 & Z_{o} \\
\frac{1}{Z_{i}} & \frac{Z_{o}}{Z_{i}}
\end{array}\right)\left(\begin{array}{c}
V_{V 2} \\
I_{V 2}
\end{array}\right) .
$$

If the impedance meter with the added front-end is connected to the impedance under test $\left(Z_{x}\right)$ through a symmetric passive network as in Fig. 5, we have (6), shown at the bottom of the page, where $D_{I}$ has been replaced by $A_{I}\left(A_{I}=D_{I}\right.$ because of symmetry). Equation (6) leads to

$$
I_{I 1}=\frac{1}{k}\left[C_{I}+A_{I}\left(\frac{1}{Z_{x}}+\frac{1}{Z_{i}}\right)\right]\left(V_{V 2}+Z_{o} I_{V 2}\right)
$$

The measured impedance value is

$$
Z_{x m}=\frac{V_{V 2}}{I_{I 1}}=\frac{1}{\frac{1}{k}\left[C_{I}+A_{I}\left(\frac{1}{Z_{x}}+\frac{1}{Z_{i}}\right)\right]}-Z_{o} \frac{I_{V 2}}{I_{I 1}}
$$

Solving for $Z_{x}$ yields

$$
Z_{x}=\frac{1}{\alpha} \frac{1}{\frac{1}{Z_{x m}+\gamma}-\frac{\beta}{\alpha}}
$$

where

$$
\begin{aligned}
\alpha & =\frac{k}{A_{I}} \\
\beta & =\frac{C_{I}}{A_{I}}+\frac{1}{Z_{i}} \\
\gamma & =Z_{o} \frac{I_{V 2}}{I_{I 1}}
\end{aligned}
$$

and $Z_{x m}$ is the impedance measurement result obtained by the instrument (meter reading). 


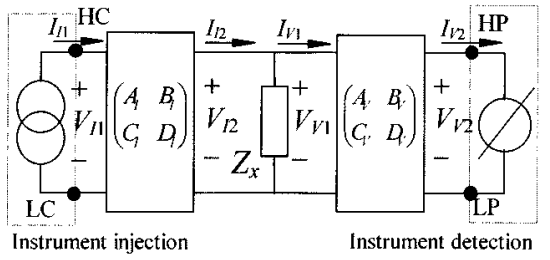

Fig. 5. Setup for impedance measurement when considering an active front-end and additional passive connecting networks.

Therefore, because $Z_{x}$ depends on three parameters $(\alpha, \beta, \gamma)$, three independent measurements can, in principle, characterize the system made from the impedance meter, the front-end amplifier, and additional connections (port extension and test fixture) without requiring the knowledge of their particular parameters. Three independent measurements yield three equations that can be solved for $\alpha, \beta$ and $\gamma$. Because (9) depends on $Z_{x m}$ (which obviously depends on $Z_{x}$ ), residual compensation in measurements involving a large range of impedance values may not be possible with a single set of three reference impedance measurements. Rather, one of the reference measurements must be chosen close to the expected value for the impedance under test ("load" value).

\section{EXPERIMENTAL RESULTS AND DISCUSSION}

The proposed solution for residual compensation has been tested in the $10 \mathrm{~Hz}$ to $10 \mathrm{MHz}$ frequency range on a system formed by an HP4192A impedance meter and a front-end amplifier like that described in [5]. Residual impedances from the front-end, port extension, and test fixtures have been measured together with the material or component under test and therefore they should be removed by the three-reference correction procedure.

The first impedance tested was a $24 \Omega$ resistor directly connected to the impedance meter. Curve A in Fig. 6 shows the result, which agrees with the specified uncertainty for the instrument. The increase in impedance around $5 \mathrm{MHz}$ is attributable to the parasitic inductance of resistor leads. Next, the $24 \Omega$ resistor was connected to four equal impedances of $39 \Omega$ in series with $4.0 \mu \mathrm{F}$ that simulate contact impedances such as those from electrodes immersed in an aqueous electrolyte. Curve B shows the result, indicating that the contact impedances increase the measurement error well above instrument specification, as expected from Fig. 3.

Then, the resistor with the added contact impedances was connected to the impedance meter through the front-end amplifier and necessary cables. Curve $\mathrm{C}$ in Fig. 6 shows that the accuracy improves as compared to curve $\mathrm{B}$, but there are still residual inaccuracies because of the unknown characteristics of the front-end amplifier and the port extensions.

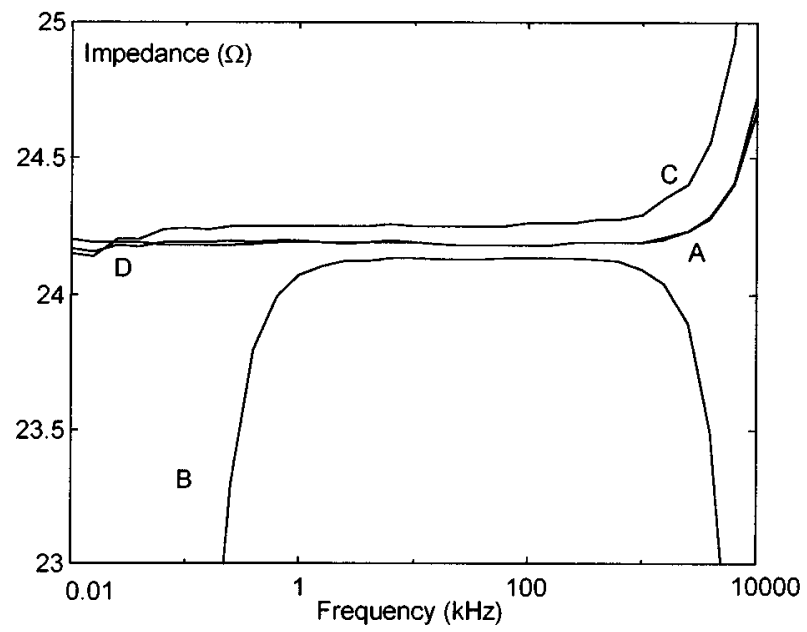

Fig. 6. Results obtained with the HP4192A impedance meter and the active front end in [5] when measuring a $24 \Omega$ resistor. (A) Direct measurement of the resistor. (B) Result when the $24 \Omega$ resistor is connected to the impedance meter using four impedances consisting of a $39 \Omega$ resistor in series with a 4.0 $\mu \mathrm{F}$ capacitor, which simulate electrode contact impedances. (C) Result when the $24 \Omega$ resistor connected to the four contact impedances is connected to the impedance meter through a front-end amplifier. (D) Calculated result after applying the residual correction procedure to the impedance displayed by the instrument

The next step was to apply the procedure for compensating residual impedances described by (9). The $Z_{x m}$ values were the meter readings at each frequency (curve $\mathrm{C}$ in Fig. 6). The three reference impedances were $1 \Omega, 120 \mathrm{k} \Omega$, and $24 \Omega$ resistors. The $1 \Omega$ resistor was close to the short-circuit impedance (a true 0 $\Omega$ impedance is impossible to achieve in practice). The $120 \mathrm{k} \Omega$ resistor was large enough as compared to the impedance under test to be considered "open circuit;" the impedance meter would not work under actual open-circuit connection. The $24 \Omega$ reference resistor was selected because it was known in advance to be close to the impedance under test. Curve D in Fig. 6 shows that the calculated final results are very close to those for the direct measurement of the $24 \Omega$ resistor, meaning that the proposed procedure actually compensates for residual impedances in the frequency range considered.

The proposed compensation method is not exclusive of any particular front-end circuit, provided it can be described by four transmission parameters as in Fig. 5. Hence, the gain and the input and output impedance of the front-end amplifier in Fig. 4 may be different from those of the unit used here. The best results are obtained when the third reference measurement is close to the expected impedance result.

This residual correction method is particularly convenient for impedance measurements involving electrodes immersed in electrolytes. We have measured a $0.9 \% \mathrm{NaCl}$ saline solution in a metacrylate cell $\left(0.4 \mathrm{~cm}^{-1}\right.$ cell constant $)$ using four stainless steel electrodes with $0.94 \mathrm{~cm}^{2}$ contact area. The theoretical

$$
\left(\begin{array}{l}
V_{I 1} \\
I_{I 1}
\end{array}\right)=\frac{1}{k}\left\{\begin{array}{ll}
A_{I}+B_{I}\left(\frac{1}{Z_{x}}+\frac{1}{Z_{i}}\right) & Z_{o}\left[A_{I}+B_{I}\left(\frac{1}{Z_{x}}+\frac{1}{Z_{i}}\right)\right] \\
C_{I}+A_{I}\left(\frac{1}{Z_{x}}+\frac{1}{Z_{i}}\right) & Z_{o}\left[C_{I}+A_{I}\left(\frac{1}{Z_{x}}+\frac{1}{Z_{i}}\right)\right]
\end{array}\right\}\left(\begin{array}{c}
V_{V 2} \\
I_{V 2}
\end{array}\right)
$$




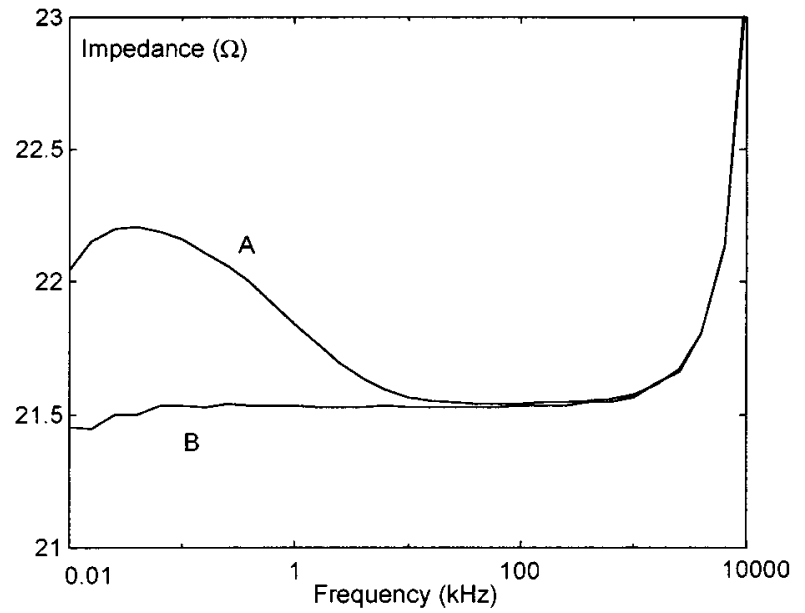

Fig. 7. Results from a measurement cell with a $0.9 \% \mathrm{NaCl}$ saline solution. (A) Impedance reading when using a front-end amplifier but no residual impedance correction. (B) Calculated impedance when using open/short/load correction.

value of the bulk impedance was about $25 \Omega$ resistive at room temperature [7]. According to Geddes [1], the approximate equivalent electrode impedance for stainless steel electrodes immersed in saline water is about $37 \Omega$ in series with $4.0 \mu \mathrm{F}$ at $1 \mathrm{kHz}$. Anyway, the final result should not depend on electrode impedance.

Curve A in Fig. 7 is the reading of the impedance meter when measuring the cell full of $0.9 \%$ saline using a front-end amplifier. Because of the high-contact impedance of the electrodes, there are large residual inaccuracies, mainly at low frequency.

Next, the procedure for correcting residual impedances described by (9) was applied by measuring the impedance of the "short circuit" and "open circuit" cell and that of a reference 25 $\Omega$ resistor (with ohmic contact, not with electrodes). The "short circuit" cell was a cell full of a saturated saline solution. The "open circuit" cell was an empty cell. Curve B in Fig. 7 shows that the open/short/load correction procedure compensates the impedance residuals at low frequencies, as needed.

An apparent shortcoming of the compensation procedure described is the need of a reference measurement close to the impedance under test, which is unknown by definition. However, curve $\mathrm{C}$ in Fig. 6 and curve $\mathrm{A}$ in Fig. 7 provide a clue for the value sought. Hence, the whole correction procedure is better composed of two steps. The first step is to measure the unknown impedance by using the external front end without any correction in order to obtain a first estimate of its value. The second step is to measure the "short circuit" and "open circuit" impedances and a known impedance close to the above estimate in the first step. Equation (9) yields then the closest estimate of the impedance under test.

\section{CONCLUSION}

Some commercial impedance meters based on auto-balancing techniques cannot be directly applied to impedance measurements in ionic media because of the high-contact impedance of the electrodes involved. Those impedance meters, offering separate current injection and voltage detection terminals (four-wire measurements), can measure the impedance of ionic media by adding a high-input impedance external preamplifier. The residual impedances of the connecting cables and front-end amplifier can then be compensated by measuring at least three reference impedances. One of these impedances must be preferably close in value to the unknown impedance. The front-end and the residual correction procedure here described open new application fields to these impedance meters that otherwise could not be applied to electrolytes.

\section{REFERENCES}

[1] L. A. Geddes, "Electrodes," in Principles of Applied Biomedical Instrumentation, 3rd ed. New York: Wiley, 1989, ch. 9.

[2] B. J. Christensen, R. T. Coverdale, R. A. Olson, S. J. Ford, E. J. Garboczi, H. M. Hennings, and T. O. Mason, "Impedance spectroscopy of hydrating cement-based materials: Measurement, interpretation and application," J. Amer. Ceram. Soc., vol. 77, no. 11, pp. 2789-2804, 1994.

[3] J. R. Macdonald, Impedance Spectroscopy, Emphasizing Solid Materials and Systems, J. R. Macdonald, Ed. New York: Wiley, 1987.

[4] M. Honda, The Impedance Measurement Handbook. A Guide to Measurement Technology and Techniques. Tokyo, Japan: Yokogawa-Hewlett-Packard, 1989.

[5] E. Gersing, "Measurement of electrical impedance in organs. Measuring equipment for research and clinical applications," Biomed. Tech., vol. 36, pp. 6-11, 1991.

[6] "Effective Impedance Measurement Using OPEN/SHORT/LOAD Correction," Hewlett Packard, Application Note 346-3, HP Literature no. 5091-6553E.

[7] A. Stogryn, "Equations for calculating the dielectric constant of saline water," IEEE Trans Microwave Tech., vol. MTT-19, pp. 733-736, Aug. 1971.

Josep M. Torrents received the Enginyer de Telecomunicació and Doctor Enginyer de Telecomunicació degrees in 1989 and 1996 respectively, both from the Universitat Politècnica de Catalunya, Barcelona, Spain.

$\mathrm{He}$ is an Associate Professor of Electronic Engineering at the Universitat Politècnica de Cataluny, and he teaches courses in several areas of electronic instrumentation. From 1998 to 1999, he was a Visiting Scholar at Northwestern University, Evanston, IL. His research includes instrumentation methods based on electrical impedance measurements, mainly applied to soil, ceramic materials, and oils.

Ramon Pallàs-Areny (F'98) received the Ingeniero Industrial and Doctor Ingeniero Industrial degrees from the Universitat Politècnica de Catalunya, Barcelona, Spain, in 1975 and 1982, respectively.

$\mathrm{He}$ is a Professor of electronic engineering at the Universitat Politècnica de Catalunya, and he teaches courses in medical and electronic instrumentation. In 1989 and 1990, he was a Visiting Fulbright Scholar and, in 1997 and 1998, he was an Honorary Fellow at the University of Wisconsin, Madison. In 2001, he was nominated Professor Honoris Causa by the Faculty of Electrical Engineering of the University of Cluj-Napoca, Romania. His research includes instrumentation methods and sensors based on electrical impedance measurements, sensor interfaces, ECG and arterial blood pressure measurements, and electromagnetic compatibility in electronic systems. He is the author of several books on instrumentation in Spanish and Catalan, the latest one being Sensors and Interfaces, Solved Problems (Barcelona, Spain: Edicions UPC, 1999). He is also coauthor (with John G. Webster) of Sensors and Signal Conditioning (2nd ed., New York: Wiley, 2001), and Analog Signal Processing (New York: Wiley, 1999).

Dr. Pallàs-Areny was a recipient, with John G. Webster, of the 1991 Andrew R. Chi Prize Paper Award from the IEEE Instrumentation and Measurement Society. He is a member of the Biomedical Engineering Society and the International Society for Measurement and Control. 